\title{
Rapid response teams: a diagnostic dilemma
}

\section{Andre Carlos Kajdacsy-Balla Amaral, ${ }^{1,2}$ Hannah Wunsch ${ }^{3,4}$}

The implementation of rapid response teams (RRTs), also known as medical emergency teams, across the world has happened in parallel with the research to assess their effectiveness. ${ }^{1}$ The development of RRTs occurred due to observations that associated signs and symptoms are often present hours or days before clear clinical deterioration in the majority of patients. ${ }^{2}$ By assessing these patients early, RRTs would presumably prevent progression to cardiopulmonary arrest.

The article by Akhtar et al assesses the performance of a RRT in three NHS Acute Hospitals in England within a single NHS Trust, with a particular focus on the variability in duration and diagnostic accuracy of the call placed to trigger the RRT. ${ }^{3}$ Examining 426 RRT activations, the authors identified significant variation in the duration of the call placed, with the call taking anywhere from $6 \mathrm{~s}$ to $92 \mathrm{~s}$. The authors then examined the recording to identify the causes of a longer call time, concluding that a substantial source of delay was confusion over whether to identify a situation as a medical emergency or a cardiac arrest. This leads to the question of whether RRTs should act separate from, or as

\footnotetext{
${ }^{1}$ Department of Critical Care Medicine, Sunnybrook Health Sciences Centre, Toronto, Ontario, Canada; ${ }^{2}$ Interdepartmental Division of Critical Care Medicine and Department of Medicine, University of Toronto, Toronto, Ontario, Canada; ${ }^{3}$ Department of Anesthesiology, Columbia University, New York, New York, USA; ${ }^{4}$ Department of Epidemiology, Columbia University, New York, New York, USA
}

Correspondence to Dr Andre Carlos Kajdacsy-Balla Amaral, Sunnybrook Health Sciences Centre, 2075 Bayview Ave, Office D1 34, Toronto, ON, Canada M4N 3M5; andrecarlos.amaral@sunnybrook.ca an extension of, 'code' teams, which primarily focus on resuscitation of patients who have already had a cardiac arrest.

Early studies of RRTs focused on decreasing the frequency of cardiac arrests, suggesting that RRTs were originally created to only respond to a medical emergency before the onset of an arrest. Most of these data came from single-centre studies that used a 'before-after' design, comparing outcomes for the patients cared for before implementation of the RRT with outcomes after implementation. $^{4-7}$ But, a large multicentre cluster-randomised trial failed to show benefits in the rates of cardiac arrest after the introduction of RRTs. ${ }^{8}$ The most obvious explanation for this difference in findings is that the early studies did not have contemporary controls ${ }^{9}$ and were influenced by trends unrelated to the implementation of the RRTs. The alternative explanation is that incredible variation exists in the healthcare systems and in the structure of the RRTs implemented in different studies, resulting in different efficacies.

As in many aspects of medicine, and particularly in critical care, where there is uncertainty there is variability. Regardless of whether a RRT exists in a hospital, there is large variability in many aspects of critical care that may impact how a RRT fits in. First, we know that the availability of intensive care unit (ICU) beds varies markedly across countries. ${ }^{10} 11$ The thresholds for admission to ICU also vary. For example, data from comparisons between the USA and the UK show that while roughly $70 \%$ of medical patients in the UK are mechanically ventilated within the first $24 \mathrm{~h}$ of admission to ICU, and have a high severity of illness, in the USA only $30 \%$ receive mechanical ventilation and the average severity of illness is substantially lower. ${ }^{12}$ The roles of RRTs are likely to be very different in these two systems. With a lower threshold for ICU admissions, such as in the USA, RRTs may be relevant in providing a timely initial assessment and subsequent communication with the ICU for transfer; while in a more restrictive system, where decisions regarding ICU triage become more relevant, RRTs may be a fundamental resource in providing initial ICU care on the ward, such as non-invasive ventilation while waiting for an ICU bed, ${ }^{13}$ or in observing a patient with severe sepsis who might be able to remain on the ward after the first $4-6 \mathrm{~h}$ of treatment.

The availability of resources to care for patients on the wards or in the ICU may also differ across countries. For example, in some Israeli hospitals, many elderly patients receive mechanical ventilation on the wards. ${ }^{14}$ In such a system, many of the ward nurses and physicians may already be familiar with critical care concepts and be less likely to need assistance from an outside team. It is therefore difficult to study the effect size of RRTs in different systems, and it is not implausible that systems that have fewer ICU beds may benefit from RRTs the most. In fact, in another trial with contemporary controls in the UK, a stepped wedge implementation of RRTs was associated with a $30 \%$ decrease in mortality in wards with RRT coverage. ${ }^{15}$

While it appears that RRTs were initially envisioned as a team separate from a cardiac arrest team, ${ }^{16}$ in some instances, such as described in the current paper, they may be combined in a single team. When a RRT plays both roles, the time to activation (measured in minutes or seconds) becomes relevant. Small delays in 
activating a RRT responding to a patient who is deteriorating are less likely to matter. However, the same is probably not true for cardiac arrest teams as the longer the time to initiation of resuscitation, the lower the likelihood of survival after cardiac arrest, ${ }^{17}$ and the differences in time to initiate treatment between survivors and non-survivors of cardiac arrests can be very small, in the range of 1-2 min. In the current study, the total delay after identification of a cardiac arrest by the healthcare providers averaged $72 \mathrm{~s}$, which could have significant adverse effects on patient care.

The current data suggest that ideally either response teams should be separated or no distinction should be attempted at the point of call to distinguish between an arrest and a deterioration, such that every call would be treated with maximal expedience. The direct call from the clinicians at the bedside may lack specificity (eg, there may be many calls that are not true arrests), but it is important to optimise a system to provide timely responses to true cardiac arrests.

The paper by Akhtar et al also gives interesting insights into another source of variability: the staffing of RRTs around the world. ${ }^{3}$ Not all RRTs have physicians. In fact, a survey across the UK identified a medical consultant as involved with the RRT less than a third of the time, with a conclusion that RRTs remained a nurse-based service $^{18}$; by contrast, in a similar survey in the Netherlands, $>60 \%$ of the teams had a critical care physician. ${ }^{19}$ In other locations, RRTs may include respiratory therapists, hospitalists and even pharmacists. It is unlikely that these differently staffed teams all provide the same level and type of support. This study demonstrates the many ways a RRT may vary in its staffing and response mechanisms and highlights the difficulty of implementing such teams into complex hospital systems.
Current data do not provide strong support regarding the effectiveness of RRTs. But understanding these large differences in the healthcare systems, RRT staffing models and RRT function become important for future studies. Given that RRTs are not applied as treatments for individual patients, but across a healthcare system, we can construct a parallel between diagnosing and treating a patient and diagnosing and treating a hospital. When faced with an individual patient presenting with shock, physicians need to identify the aetiology and then treat the patient accordingly. While some care measures may be non-specific and universally employed, it is ultimately important to detect whether the shock is due to pneumonia and sepsis or pericardial tamponade since the fundamental interventions will be very different. The same applies for improving healthcare systems; while there may be non-specific supportive measurements that are applied everywhere, such as cardiac arrest teams, specific decisions, such as the implementation of a RRT, should be based on a diagnostic evaluation. While a RRT may be the correct 'treatment' for deteriorating patients on the ward, some hospitals may actually find that having a hospitalist available around the clock or increasing the nurse to patient ratio may be more important first steps. Medical students are taught to describe the disease before prescribing the treatment. In our eagerness to embrace the new science of improving quality in healthcare and implementing changes, we may have jumped that fundamental step: making the diagnosis.

Competing interests None.

Provenance and peer review Commissioned; internally peer reviewed.

\section{Published Online First 18 November 2011}

BMJ Qual Saf 2012;21:177-178.

doi:10.1136/bmjqs-2011-000589

\section{REFERENCES}

1. Chan PS, Jain R, Nallmothu BK, et al. Rapid response teams: a systematic review and meta-analysis. Arch Intern Med 2010;170:18-26.

2. McQuillan $P$, Pilkington $S$, Allan A, et al. Confidential inquiry into quality of care before admission to intensive care. BMJ 1998;316:1853-8.

3. Akhtar N, Field R, Greenwood L, et al. Quality of in-hospital cardiac arrest calls: a prospective observational study. BMJ Quality \& Safety 2012;21:184-190.

4. Bellomo R, Goldsmith D, Uchino S, et al. A prospective before-and-after trial of a medical emergency team. Med J Aust 2003;179:283-7.

5. Sharek PJ, Parast LM, Leong K, et al. Effect of a rapid response team on hospital-wide mortality and code rates outside the ICU in a Children's Hospital. JAMA 2007;298:2267-74.

6. Buist M, Harrison J, Abaloz E, et al. Six year audit of cardiac arrests and medical emergency team calls in an Australian outer metropolitan teaching hospital. BMJ 2007;335:1210-12.

7. Jones D, Bellomo R, Bates S, et al. Long term effect of a medical emergency team on cardiac arrests in a teaching hospital. Crit Care 2005;9:R808-15.

8. Hillman $\mathrm{K}$, Chen J, Cretikos M, et al. Introduction of the medical emergency team (MET) system: a cluster-randomised controlled trial. Lancet 2005;365:2091-7.

9. Joffe AR, Anton NR, Burkholder SC. Reduction in hospital mortality over time in a hospital without a pediatric medical emergency team: limitations of before-andafter study designs. Arch Pediatr Adolesc Med 2011;165:419-23.

10. Wunsch $\mathrm{H}$, Angus DC, Harrison DA, et al. Variation in critical care services across North America and Western Europe. Crit Care Med 2008;36:2787-9.

11. Adhikari NK, Fowler RA, Bhagwanjee S, et al. Critical care and the global burden of critical illness in adults. Lancet 2010;376:1339-46.

12. Wunsch $\mathrm{H}$, Angus DC, Harrison DA, et al. Comparison of medical admissions to intensive care units in the United States and United Kingdom. Am J Respir Crit Care Med 2011;183:1666-73.

13. Schneider AG, Calzavacca P, Mercer I, et al. The epidemiology and outcome of medical emergency team call patients treated with non-invasive ventilation. Resuscitation 2011;82:1218-23.

14. Hersch M, Sonnenblick M, Karlic A, et al. Mechanical ventilation of patients hospitalized in medical wards vs the intensive care unit-an observational, comparative study. J Crit Care 2007;22:13-17.

15. Priestley G, Watson W, Rashidian A, et al Introducing critical care outreach: a wardrandomised trial of phased introduction in a general hospital. Intensive Care Med 2004;30:1398-404.

16. Jones DA, DeVita MA, Bellomo R. Rapidresponse teams. $N$ Engl J Med 2011;365:139-46.

17. Rade B. Survival from prehospital cardiac arrest is critically dependent upon response time. Resuscitation 2006;69:229-34.

18. McDonnell A, Esmonde L, Morgan R, et al. The provision of critical care outreach services in England: findings from a national survey. $J$ Crit Care 2007;22:212-18.

19. Ludikhuize J, Hamming A, de Jonge $E$, et al. Rapid response systems in The Netherlands. Jt Comm J Qual Patient Saf 2011;37:138-44, 97. 